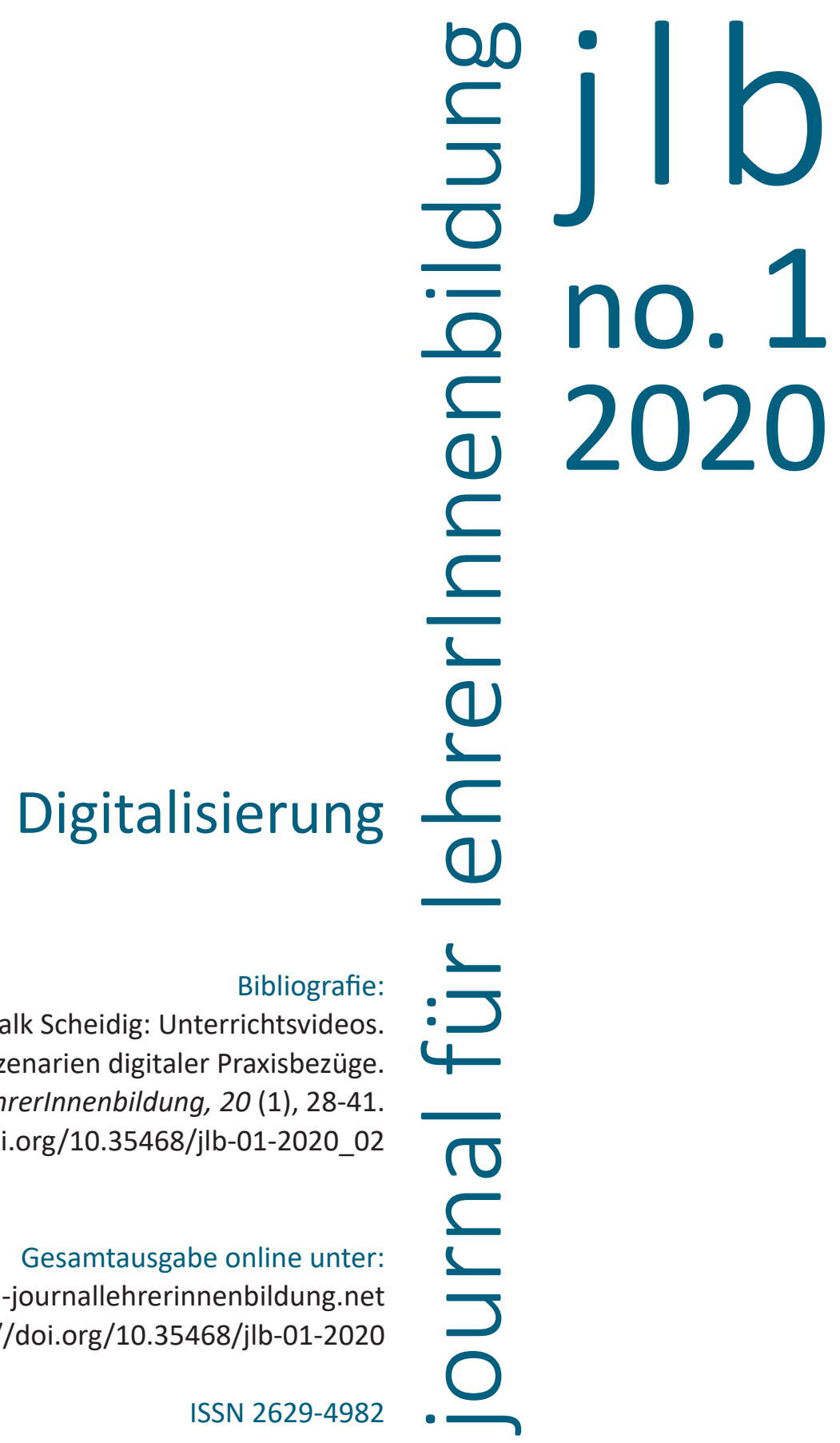


02
Falk Scheidig

\section{Unterrichtsvideos. \\ Neue Szenarien \\ digitaler Praxisbezüge}




\section{Unterrichtsvideos als Praxiszugänge}

Das Herstellen von Praxisbezügen mit dem Ziel der vorbereitenden Berufsqualifizierung ist - ungeachtet der Diskussion um adäquate Form und Intensität - ein zentrales Anliegen hochschulischer Lehrpersonenbildung. Es lassen sich graduell variierende „approximations of practice" (Seidel \& Thiel, 2017, S. 3) mit je spezifischer Ausrichtung und Anforderung differenzieren (Scheidig, 2017): von der sprach- bzw. textvermittelten Auseinandersetzung mit Praxis (z. B. mündliche und schriftliche Praxisberichte, Artefakte der Praxis wie Stundenplanungen) bis hin zum direkten Praxiskontakt (z. B. Hospitation, Forschungsprojekt in/mit der Schulpraxis, Gestaltung eigenen Unterrichts). Der Einsatz von Unterrichtsvideos, der in Lehramtsstudiengängen keineswegs neu ist, aber in den vergangenen Jahren zunahm (Petko, Prasse \& Reusser, 2014), positioniert sich auf dieser Achse zwischen den beiden Polen als zwar medienvermittelter, aber vergleichsweise authentischer Praxiszugang: „video offers pre-service teachers a ,window' into teaching without the pressure of having to interact in the classroom situation" (Blomberg, Renkl, Sherin, Borko \& Seidel, 2013, S. 93).

Einige der Vorzüge werden darin gesehen, dass Unterrichtsvideos (1) die komplexe und dynamische Unterrichtswirklichkeit hoch verdichtet und mit mehr Informationen als beispielsweise Texte erfassen, dass (2) die Kombination aus der "stellvertretenden Teilhabe an realen Unterrichtssituationen" (Gröschner, Klaß \& Dehne, 2018, S. 198) einerseits und der zeit-, orts- und handlungsdistanzierten Betrachtung andererseits verschiedene Optionen der Theorie-Praxis-Relationierung hervorbringt, dass (3) Bezüge zu konkreten Unterrichtssituationen und Herausforderungen des Lehrberufs kontextualisiert hergestellt werden (können), dass (4) im Gegensatz etwa zur Live-Beobachtung die Möglichkeit wiederholenden Betrachtens u. a. unter Nutzung von Zoom- und Zeitlupenfunktion und verschiedener visueller und fachlicher Perspektiven besteht, dass (5) die verbindliche Gegenständlichkeit auf der Sichtebene das Finden einer gemeinsamen Sprache über Unterricht befördert, dass (6) die Darstellung von Praxis nicht durch Erinnerungsvermögen, tendenziöse oder selektive Darlegung, Deutung oder textimmanente Interpretationsspielräume verzerrt wird. Wesentliche technische Hürden des Videoeinsatzes in der Lehrpersonenbildung sind gesunken, insbesondere durch die Omnipräsenz 
von mobilen Geräten, die gestiegenen Speicherkapazitäten und die Verbreitung des Internets mit erhöhten Datenübertragungstempi. Bedurfte es in den 1960er Jahren noch aufwendiger „Unterrichtsmitschauanlagen“ (Meyer \& Aulinger, 2019; Petko et al., 2014), können Studierende heute - nach entsprechender Einführung und Datenschutzklärung - mit Alltagsgeräten eigene (oder untereinander, siehe z. B. Kleinknecht \& Gröschner, 2016) Unterrichtserfahrungen aufzeichnen (Kamera oder Handy mit Stativ) sowie als Videodatei bearbeiten, abspielen (Laptop, Tablet) und in variablen Studienkontexten verwenden. Zugleich erwachsen aus zahlreichen SoftwareEntwicklungen neue Optionen der konkreten Arbeit mit Videos im Studium. Die digitale Transformation erweitert in verschiedener Hinsicht den Möglichkeitsraum für videobasierte Praxisbezüge im Studium.

Der Beitrag nimmt dies zum Ausgangspunkt und verschafft einen Überblick über neuere Szenarien des Videoeinsatzes in der Lehrpersonenbildung. Die Potenziale und Problemstellungen der Analyse von Unterrichtsvideos sowie von Videofeedback in der Aus- und Weiterbildung von Lehrpersonen sind Gegenstand zahlreicher internationaler Studien zu Effekten und Erträgen des Videoeinsatzes (für eine Übersicht: Blomberg et al., 2013; Gaudin \& Chaliès, 2015; Seidel \& Thiel, 2017). Die nachfolgenden Ausführungen beschränken sich deshalb auf die konzise Skizze und Systematisierung von Nutzungsmöglichkeiten von Unterrichtsvideos unter besonderer Gewichtung jener (digitalen) Szenarien, die auf neuere Entwicklungen verweisen und/ oder bislang noch keine weite Verbreitung in Lehramtsstudiengängen fanden.

\section{Szenarien und digitale Entwicklungen}

\section{Videos in der Präsenzlehre}

Die wohl prominenteste Form des Videoeinsatzes stellt die Einbettung von Videos in Präsenzlehrveranstaltungen dar. Dies folgt oftmals zwei Intentionen: erstens die professionelle Unterrichtswahrnehmung profilieren („,professional vision“; Sherin \& van Es, 2009), Unterricht differenziert beschreiben und Situationen theoriegestützt erklären („noticing" und „knowledge-based reasoning"; Seidel, Blomberg \& Stürmer, 2010), zweitens das fachlich begründete Denken in Handlungsoptio- 
nen und -alternativen stimulieren. Weitere Ziele können das Zeigen positiver oder kritischer Beispiele, die situierte Auseinandersetzung mit idealtypischen Situationen und Herausforderungen oder das multiperspektivische Analysieren von Unterricht mit verschiedenen fachlichen Referenzen sein (Gaudin \& Chaliès, 2015). Neben diesen professionalisierungsassoziierten Bestrebungen verbinden sich des Weiteren hochschuldidaktische Absichten mit dem Videoeinsatz, insbesondere Motivierung der Studierenden, Aktivierung von Vorwissen, Anregung dialogischer Phasen und Anregung studentischer Beiträge in der Lehrveranstaltung. Demgegenüber finden sich nur vereinzelt berichtete Aktivitäten, die direkt darauf zielen, die schulische Lehrkompetenz der Studierenden durch Videos zu erweitern bzw. Lehrverhalten optimierend zu verändern (z. B. Gröschner, Schindler, Holzberger, Alles \& Seidel, 2018). In der Regel handelt es sich bei den eingesetzten Videos um kurze, prägnante Ausschnitte (Videovignetten), die zumeist in Seminaren unter Akzentuierung ausgewählter Aspekte, teilweise aber auch mit Aufträgen im Selbststudium, beispielsweise über ein LearningManagement-System (z. B. Bartel \& Roth, 2015), eine Analyse erfahren.

\section{Video-Plattformen}

Dem Videoeinsatz in der Präsenzlehre arbeiten verschiedene, oftmals öffentlich zugängliche Online-Plattformen für die Arbeit mit Unterrichtsvideos zu, die in den vergangenen beiden Dekaden angelegt wurden (Pekto et al., 2014) und zahlreiche Unterrichtsvideos - z. B. geordnet nach didaktischen Schlagworten, Fächern, Schulstufen/ -arten und Ländern - zur Verfügung stellen (z. B. http://www. unterrichtsvideos.ch, https://www.teachingchannel.org). An der LMU München entsteht aktuell ein Portal, das erlaubt, mit mehreren Sichtachsen videografierte Unterrichtsstunden simultan aus verschiedenen Perspektiven (Lehrperson, Klasse, einzelne Schüler*innen) zu betrachten und innerhalb der Online-Umgebung eine qualitative Inhaltsanalyse mit einem dynamischen Kategoriensystem vorzunehmen (Meyer \& Aulinger, 2019). Ein Vorteil der geteilten (hochschulübergreifenden) Nutzung von Videos auf entsprechenden Plattformen liegt darin, dass diese Videos partiell mit Expert*innenratings, spezifischen Analysehinweisen und Begleitmaterial versehen sind, auf die bei der didaktischen Aufbereitung zurückgegriffen werden kann. 


\section{Social Video Learning}

Einige Video-Plattformen sind insofern als erweiterte Arbeits- und Lernumgebung konzipiert, als sie über konventionelle Abspielfunktionen hinaus virtuelle Interaktionsoptionen bieten. Hervorzuheben sind insbesondere die unter Social Video Learning gefassten Potenziale, die aus der Möglichkeit situationsgenauen Annotierens in Videos erwachsen, mit der Unterrichtsmitschnitte sowohl individuell als auch kollaborativ über zeitstempelbasierte Markierungen, Notizen und Re-Kommentierungen synchron oder asynchron auf der Plattform erschlossen werden können (Mayer-Frühwirth, 2017; Vohle, 2013). Hierfür kann auch auf nicht eigens für Unterrichtsvideos bzw. die Lehrpersonenbildung entwickelte Videoplattformen (z. B. https://edubreak.de), LMS-Plug-ins oder Standalone-Videosoftware zurückgegriffen werden (Petko et al., 2014).

Die Möglichkeit, auf (einigen) Videoplattformen eigene Unterrichtsvideos hochzuladen und zu teilen (user-generated content), verweist auf die grundlegende didaktische Differenzierung zwischen der Arbeit mit eigenen und fremden Unterrichtsvideos. Studien zeigen, dass fremde Unterrichtsvideos eher eine distanzierte Reflexion, das Kreieren von Handlungsalternativen und die Motivation der Studierenden befördern, eigene Videos hingegen eher die Identifikation und die Aktivierung von Vorwissen begünstigen (Gaudin \& Chaliès, 2015; Kleinknecht \& Gröschner, 2016; Seidel \& Thiel, 2017). Die Entscheidung zugunsten einer der beiden Varianten steht in Abhängigkeit vom Lernziel, ebenso wie die Wahl, ob "best practice", "typical practice" oder "critical incidents" fokussiert werden (Blomberg et al., 2013; Petko et al., 2014). Fremde Unterrichtsvideos können dabei videografiertes Handeln von Mitstudierenden, Unterrichtsmitschnitte unbekannter Lehrpersonen sowie geskriptete Videoaufnahmen umfassen. Videos letztgenannter Kategorie sind aufgrund des schauspielerischen Settings anspruchsvoll und kostenintensiv, aber $u$. a. deshalb von Interesse, weil sie die Hervorhebung von spezifischen Unterrichtsaspekten, die evidenzbasierte Gestaltung von modellhaften Sequenzen oder auch das Erfassen von ausgeprägt ineffektiven oder kritischen Lehr-/Lernsituationen erlauben, für die sich nicht immer authentische Videoaufnahmen finden lassen (Piwowar, Barth, Ophardt \& Thiel, 2018; Schröder \& Fischler, 2005). 


\section{Videogestütztes Online-Feedback}

Die Anfertigung und Nutzung eigener Unterrichtsvideos ist zumeist hochschuldidaktisch mit einer gegenseitigen Unterrichtsanalyse unter Peers gerahmt, also einer Kombination der Betrachtung von eigenen und fremden Unterrichtsvideos, bei der Studierende die Rolle von sog. „Prosumenten“ (Portmanteauwort aus Produzent und Konsument) übernehmen. Waren solche „video clubs" (Sherin \& van Es, 2009) früher als Präsenzveranstaltungen zur diskursiven Perspektiverweiterung angelegt, lassen sich mit den entsprechenden Webumgebungen (LMS, Videoplattform) Prozesse des Peer-Learning und PeerFeedbacks online realisieren, z. B. als Blended-Learning-Format (König, 2019) oder als synchrone, in Präsenzveranstaltungen implementierte Videoarbeit mit eigenen mobilen Geräten für Gruppen- oder individuelle Arbeitsformen. Die Potenziale virtuell-asynchroner Videoarbeit entfalten sich z. B. in Praxisphasen und -semestern, in denen Studierende nur zeitlich eingeschränkt oder stark zeitversetzt die Möglichkeit besitzen, sich angeleitet und strukturiert mit Kommiliton*innen über konkrete Unterrichtserfahrungen auszutauschen. Für Dozierende bzw. Mentor*innen erwächst hieraus zudem die Möglichkeit, auf Basis studentischer Unterrichtsaufzeichnen, die mit ihnen geteilt werden, ein ortsunabhängiges formatives Feedback bzw. Online-Coaching im Sinne einer systematischen Begleitung in Praktika zu geben, um deren Ertrag für die individuelle Professionalisierung zu steigern (Gröschner, Klaß \& Dehne, 2018). Kleinknecht und Gröschner (2016) berichten gestützt auf eine Prä-Post-Kontrollgruppenstudie von einer positiven Wirkung virtuellen Videofeedbacks und einer Zunahme der Reflexionstiefe. Das zugrundeliegende didaktische Design verzahnt als digitaler "Video Feedback Cycle" sequentiell die folgenden Elemente: die schriftliche Reflexion eines selbstgewählten, annotierten und mit Material angereicherten Ausschnitts einer eigenen Unterrichtsstunde, das Peer-Feedback von Mitstudierenden über eine Online-Plattform zum Video und der Selbstreflexion, zwei hierauf bezogene Feedbacks von Lehrerbildner*innen und das Verfassen einer zweiten Selbstreflexion auf Basis der multiperspektivischen Rückmeldungen.

\section{E-Portfolio mit Videos}

Für die Kombination der Videoarbeit mit individuell-reflexiven Zugängen bieten sich insbesondere auch multimediale, online-geführte 
E-Portfolios an, die es gestatten, die analytische, evaluative und entwicklungsorientierte Auseinandersetzung mit dem eigenen Professionalisierungsprozess gezielt mit Material verschiedenen Typs wie etwa Unterrichtsvideos anzureichern. E-Portfolio-Software wie Mahara gewährt zudem die Möglichkeit, die Reflexion bzw. Dokumentsammlung für Dozierende oder Mitstudierende zur Online-Kommentierung zu öffnen. Eine Weiterentwicklung stellen mobile E-Portfolios dar - z. B. die App metapholio, mit der Studierende in unmittelbarer zeitlicher Nähe zu relevanten Praxissituationen mit ihrem Smartphone oder Tablet Eindrücke in Textform, als Fotos, Audionotizen oder eben Videos festhalten, in ihr E-Portfolio integrieren und mit anderen Personen teilen können (Petko, Schmid, Müller \& Hielscher, 2019).

\section{Videos als Prüfungsstimulus}

Die zuvor referierten Ansätze verbindet ihr formativer Charakter. Daneben existieren Beispiele für die Einbettung von Videos für summative Kompetenzbilanzierungen. Ein Beispiel ist die Nutzung von Videos entweder als Stimulus für eine praxisnahe Wissensentfaltung zur Demonstration von relevanten Kompetenzen wie professionelle Wahrnehmung, begriffliche Fassung, wissensbasierte Analyse und Beurteilung von Praxisphänomenen, Entwurf und Explikation von Handlungsoptionen, problemorientierte Fallarbeit usw. (Kersting, Givvin, Thompson, Santagata \& Stigler, 2012). Das Spektrum potenzieller Modi der Einbettung von Unterrichtsvideos in Leistungsnachweise ist breit und erstreckt sich von mündlichen Gruppen- und Einzelprüfungen über offene und geschlossene Klausuren bis hin zu schriftlichen Erarbeitungen wie Hausarbeiten und Essays. Eine mögliche technische Lösung zur Umsetzung liefern computergestützte Prüfungen (E-Assessments), bei denen videozentrierte Aufgaben individuell - im Falle von Präsenzprüfungen: am CIP- oder Mobilgerät mit Kopfhörern - in einer geschlossenen Softwareumgebung (kiosk mode) oder in einem offenen Format (open book exam) einbezogen werden können.

\section{Videoportfolio als Leistungsnachweis}

Im Hinblick auf die Nutzung von Unterrichtsvideos zu Prüfungszwecken ist der Kompetenznachweis an einem Video zu unterscheiden von der performativen Dokumentation der Kompetenzentwicklung 
in einem Video. Dergestalt angelegte Leistungsnachweise zentrieren sich auf die professionelle Unterrichtstätigkeit als Outcome des Lehramtsstudiums und zielen darauf, die von Studierenden entwickelte Lehrkompetenz mittels Unterrichtsvideos summativ zu beurteilen. Prominentestes Beispiel ist edTPA, ein aktuell in über 900 Lehrer*innenbildungsprogrammen in 41 US-Staaten eingesetztes Instrument (http://edtpa.aacte.org/state-policy), das sich als fach- und stufenspezifisches Portfolio am Studienende aus selbst aufgezeichneten, unbearbeiteten Unterrichtsvideos, Unterrichtsplanungs- und weiteren Dokumenten zusammensetzt und von geschulten Expert*innen für 27 verschiedene "teaching fields" respektive "content areas" nach standardisierten Kriterien bewertet wird (für ein vergleichbares Verfahren im deutschsprachigen Raum vgl. Fraefel, Bäuerlein \& Barabasch, 2018). Die videogestützte Selbstdokumentation von Lehrkompetenz wird gegenüber tradierten Unterrichtsbesuchen/Lehrproben aus verschiedenen Gründen im Vorteil gesehen (ebd.), obschon sich auch bei videografierter Unterrichtsperformanz die (Validitäts-)Frage stellt, wie prüfungsseitig mit der empirisch nachweisbaren (Praetorius, Pauli, Reusser, Rakoczy \& Klieme, 2014) intraindividuellen Instabilität von Lehrkompetenz (-komponenten) in der Frühphase eigener Unterrichtsverantwortung umzugehen ist.

\section{Eye-Tracking}

Ein wenig verbreiteter Praxiszugang ist der Einsatz von Eye-Tracking zur Erfassung unterrichtlicher Aktivitäten und Wahrnehmungen. Mit mobilen Eye-Tracking-Brillen aufgezeichnete Unterrichtsvideos erlauben einerseits, den Unterricht aus der Lehrendenperspektive nachzuvollziehen. Wie Erhebungen zeigen, führt das von konventionellen Unterrichtsvideos abweichende Sichtfeld in der videobasierten Aus- und Weiterbildung von Lehrpersonen zu Verschiebungen in der Erschließung der (eigenen) Unterrichtsvideos weg von der tendenziell dominierenden lehrer*innenzentrierten Kognition hin zu einer stärker am Lernprozess der Schüler*innen orientierten Betrachtung des Unterrichts (Cortina et al., 2018). Andererseits können mittels dieser Videos die getrackten Blickbewegungen und -fixationen der (angehenden) Lehrperson Sichtbarkeit erlangen und beispielsweise als Bezugspunkt für Feedback und die Auseinandersetzung mit professioneller Wahr- 
nehmung von Unterricht $-z$. B. im Kontext von Classroom Management - fungieren. Eine potenzielle Quelle für Unterrichtsfeedback stellen auch Unterrichtsaufnahmen aus der Perspektive der Schüler*innen dar, die aus Kosten- und Praktikabilitätsgründen auch mit unscheinbaren kamerabesetzten Brillen ohne Eye-Tracking-Funktion oder mit Kopfkameras aufgezeichnet werden können. Eye-Tracking-Hardware kann ferner eingesetzt werden, um die Perzeption authentischen Unterrichtsgeschehens auch außerhalb konkreter Praxissituationen einer Analyse zugänglich zu machen: An der Videoausgabequelle positionierte Remote Eye-Tracker ermöglichen ein blickzentriertes Erfassen der Unterrichtswahrnehmung am Monitor, um offenzulegen, welche Areale im Klassenzimmer bei der Videobetrachtung wann und wie lange verfolgt wurden - als Impulse zur Thematisierung und Weiterentwicklung professioneller Unterrichtswahrnehmung (van den Bogert, van Bruggen, Kostons \& Jochems, 2014).

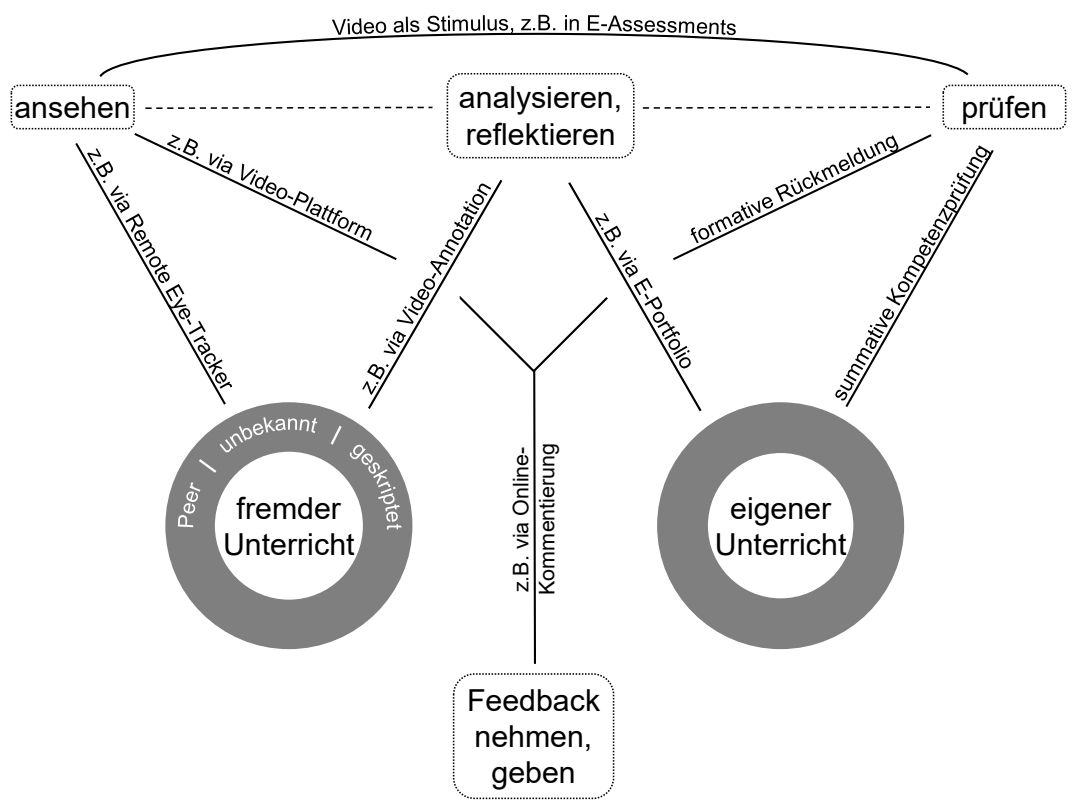

Abb. 1 Mapping ausgewählter digitaler Szenarien der Videonutzung in der Lehrpersonenbildung 


\section{Einordnung und Ausblick}

Die Szenarien stehen in unterschiedlichem Verhältnis zur Unterrichtspraxis und zueinander (Abb. 1), lassen sich verzahnen (z. B. die technische Verknüpfung von Videoannotationstool mit E-PortfolioPlattform, vgl. Grubesic, Bauer, Himpsl-Gutermann \& Meissl-Egghart, 2018) und sie können verschiedene Lehr-/Lernsettings (online/analog, Einzel-/Tandem-/Gruppen-/Plenararbeit usw.) und hochschuldidaktische Ansätze (forschendes Lernen, problembasiertes Lernen, rekonstruktive Fallarbeit, Kompetenzorientierung usw.) fundieren. In mehreren Studien wurden positive Effekte der studienintegrierten Videoarbeit in Bezug auf die Reflexionsleistung, Unterrichtswahrnehmung, Motivation und Zufriedenheit der Studierenden nachgewiesen (Gaudin \& Chaliès, 2015). Mit der Expansion und Ausdifferenzierung der Videoarbeit, die hier in groben Konturen nachgezeichnet wurde, dürften die Potenziale didaktisch wie empirisch weiter erschlossen werden, sodass neue und vertiefende Erkenntnisse zur Wirkung von Videos in Lehramtsstudiengängen zu erwarten sind. Zugleich verbindet sich mit dem Videoeinsatz ein wachsendes Konvolut an Fragen (Seidel \& Thiel, 2017), etwa hinsichtlich der erforderlichen Videoqualität, verzerrenden Reaktivitätseffekten bei der Videoerstellung, der Auswahl von geeigneten Videos und Ausschnitten, der angemessenen Ausstattung der Videos mit spezifischen Kontextinformationen, einer Überakzentuierung technischer Aspekte zuungunsten des Inhaltlichen, einer nicht-intendierten Aufmerksamkeitsverschiebung hin zur Oberflächenstruktur von Unterricht und generell der Reflexionstiefe der Videoarbeit, der Distanz zwischen konkreter Unterrichtspraxis und ihrer medialen Repräsentation im Video z. B. in Bezug auf das Erleben situativer Unterrichtsfaktoren, des Umgangs mit Domänenspezifika und fachdidaktischen Singularitäten in einer zumeist fachübergreifenden Videoarbeit, des Beitrags videogestützter Zugänge im interdisziplinären Gesamtensemble des Lehramtscurriculums usw.

Wenngleich neuere Szenarien videogestützten Praxisbezugs vor allem auf digitale Lehr-, Lern- und Arbeitsformen verweisen, besitzen sie Bezugspunkte in der Präsenzlehre und bedürfen einer professionellen Instruktion und Begleitung. Die Notwendigkeit, die Videoarbeit sowohl didaktisch als auch konzeptionell begründet einzubetten also insbesondere auf die mikrodidaktische Anlage, das Professiona- 
lisierungsverständnis und die Studienziele rekurrierend -, darf im einschlägigen Diskurs als unbestritten gelten und subordiniert das Neomanie-verdächtige Medium in Relation zu seiner Funktion für den studentischen Kompetenzerwerb (Blomberg et al., 2013). Bedeutsam scheint insbesondere die Herstellung von Kohärenz in der Bezugnahme auf Unterrichtspraxis im Studium, wobei einige der benannten Szenarien der angestrebten Kohärenz in der Lehrpersonenbildung zuzuarbeiten vermögen, z. B. wenn ein und dasselbe Unterrichtsvideo in (disziplinär) verschiedenen Modulen referenziert wird, wenn videobasierte E-Portfolios studienbegleitend und studienbereichsübergreifend geführt werden, wenn Video-Seminare oder Video-Assessments von Vertreter*innen der Fachdidaktiken und Erziehungs-/ Bildungswissenschaften in Zusammenarbeit gestaltet werden. Nicht zu unterschätzen ist das Potenzial, mit Eigenvideos der Studierenden in denen sie ihre Praxiserfahrungen dokumentieren - die oftmals isolierten Praxisbezüge der schulpraktischen Übungen respektive berufspraktischen Studien für andere Studienbereiche und -phasen zu öffnen, z. B. für ein fachdidaktisches Coaching (Schröder \& Fischler, 2005) oder die fallbasierte Theorie-Praxis-Verknüpfung im allgemeinpädagogischen Teil des Studiums.

Digitale Szenarien der Videoarbeit, wie sie im Vorangegangenen benannt wurden, dürfen nicht Selbstzweck sein, sondern legitimieren sich über die (ihnen zugedachte) Funktion in Professionalisierungsprozessen im Lehramtsstudium. Sollte ihr Einsatz aber dazu beitragen, dass angehende Lehrpersonen aufgrund des Kontakts mit digitalen Lehr-/Lernformaten ebendiesen künftig aufgeschlossen(er) gegenüberstehen und digitale Handlungsfähigkeit erwerben, dürfte dies gleichwohl ein willkommener Nebeneffekt sein - nicht zuletzt angesichts des im internationalen Vergleich geringen Digitalisierungsgrads deutschen Schulunterrichts (Drossel, Eickelmann, Schaumburg \& Labusch, 2019). Es bedürfte hierzu, wie auch grundlegend zu den Effekten digitaler Videoarbeit, eingehender Forschung, die wiederum eine mehr als nur punktuelle Implementation voraussetzt. Die Potenziale neuer Zugänge entfalten sich (ebenso wie die Sensibilisierung für damit verbundene Probleme und Limitationen) mit ihrer Nutzung, die im Falle der digitalen Videoarbeit trotz gesunkener Hürden und neuer technischer Möglichkeiten organisatorisch und didaktisch anspruchsvoll bleibt. 


\section{Literatur}

Bartel, M.-E. \& Roth, J. (2015): Diagnostische Kompetenz durch Videovignetten fördern. In F. Caluori, H. Linneweber-Lammerskitten \& C. Streit (Hrsg.), Beiträge zum Mathematikunterricht 2015 (S. 1033-1036). Münster: WTM-Verlag.

Blomberg, G., Renkl, A., Sherin, M. G., Borko, H. \& Seidel, T. (2013). Five researchbased heuristics for using video in pre-service teacher education. Journal for educational research online, 5 (1), 90-114.

Cortina, K. S., Müller, K., Häusler, J., Stürmer, K., Seidel, T. \& Miller, F. (2018). Feedback mit eigenen Augen: Mobiles Eyetracking in der Lehrerinnen- und Lehrerbildung. Beiträge zur Lehrerinnen- und Lehrerbildung, 36 (2), 208-222.

Drossel, K., Eickelmann, B., Schaumburg, H. \& Labusch, A. (2019). Nutzung digitaler Medien und Prädiktoren aus der Perspektive der Lehrerinnen und Lehrer im internationalen Vergleich. In B. Eickelmann, W. Bos, J. Gerick, F. Goldhammer, H. Schaumburg, K. Schwippert, M. Senkbeil \& J. Vahrenhold (Hrsg.), ICILS 2018 \#Deutschland. Computer- und informationsbezogene Kompetenzen von Schülerinnen und Schülern im zweiten internationalen Vergleich und Kompetenzen im Bereich Computational Thinking (S. 205-240). Münster: Waxmann.

Fraefel, U., Bäuerlein, K. \& Barabasch, A. (2018). Assessing teacher candidates' professional competence for evaluating teacher education programs: The case of German-speaking Europe. In V. C. X. Wang (Ed.), Handbook of Research on Program Development and Assessment Methodologies in K-20 Education (pp. 418-442). Hershey: IGI Global.

Gaudin, C. \& Chaliès, S. (2015). Video viewing in teacher education and professional development: A literature review. Educational Research Review, 16, 41-67. https://doi.org/10.1016/j.edurev.2015.06.001

Gröschner, A., Klaß, S. \& Dehne, M. (2018). „Praxis digital“. Einsatz neuer MedienTools im Praxissemester am Beispiel des onlinebasierten Videofeedbacks. In M. Rothland \& I. Biederbeck (Hrsg.), Praxisphasen in der Lehrerbildung im Fokus der Bildungsforschung (S. 197-207). Münster: Waxmann.

Gröschner, A., Schindler, A.-K., Holzberger, D., Alles, M. \& Seidel, T. (2018). How systematic video reflection in teacher professional development regarding classroom discourse contributes to teacher and student self-efficacy. International Journal of Educational Research, 90, 223-233.

Grubesic, K., Bauer, R., Himpsl-Gutermann, K. \& Meissl-Egghart, G. (2018). Ich sehe was, was du nicht siehst: Videoreflexion im digitalen Raum. Ein Praxisbericht. In B. Getto, P. Hintze \& M. Kerres (Hrsg.), Digitalisierung und Hochschulentwicklung. Proceedings zur 26. Tagung der Gesellschaft für Medien in der Wissenschaft (S. 222-233). Münster: Waxmann.

Kersting, N. B., Givvin, K. B., Thompson, B. J., Santagata, R. \& Stigler, J. W. (2012). Measuring Usable Knowledge: Teachers' Analyses of Mathematics Classroom Videos Predict Teaching Quality and Student Learning. American Educational Research Journal, 49 (3), 568-589.

Kleinknecht, M. \& Gröschner, A. (2016). Fostering preservice teachers' noticing with structured videofeedback: Results of an online- and video-based intervention study. Teaching and Teacher Education, 59, 45-56.

König, C. M. (2019). Peervideofeedback. Ein Blended-Learning-Konzept in der ersten Phase der Lehrer*innenbildung. In S. Robra-Bissantz, O. J. Bott, N. Kleinefeld, 
K. Neu \& K. Zickwolf (Hrsg.), Teaching Trends 2018. Die Präsenzhochschule und die digitale Transformation (S. 113-120). Münster: Waxmann.

Mayer-Frühwirth, G. (2017). Social Video Learning. Kollaboratives Reflektieren in den Schulpraktika. In C. Fridrich, G. Mayer-Frühwirth, R. Potzmann, W. Greller \& R. Petz (Hrsg.), Forschungsperspektiven 9 (S. 69-76). Münster: LIT.

Meyer, R. \& Aulinger, J. (2019). UnterrichtOnline.org - ein Videografieportal für alle Phasen der LehrerInnenbildung. Interaktives und kollaboratives webbasiertes Lehren und Lernen mit Unterrichtsvideos. In N. Pinkwart \& J. Konert (Hrsg.), DELFI 2019 - Die 17. Fachtagung Bildungstechnologien (S. 331-332). Bonn: Gesellschaft für Informatik.

Petko, D., Prasse, D. \& Reusser, K. (2014). Online-Plattformen für die Arbeit mit Unterrichtsvideos: Eine Übersicht. Beiträge zur Lehrerinnen- und Lehrerbildung, 32 (2), 247-261.

Petko, D., Schmid, R., Müller, L. \& Hielscher, M. (2019). Metapholio: A Mobile App for Supporting Collaborative Note Taking and Reflection in Teacher Education. Technology, Knowledge and Learning, 24, 699-710. https://doi.org/10.1007/s10758019-09398-6

Piwowar, V., Barth, V. L., Ophardt, D. \& Thiel, F. (2018). Evidence-based scripted videos on handling student misbehavior: the development and evaluation of video cases for teacher education. Professional Development in Education, 44 (3), 369-384.

Praetorius, A.-K., Pauli, C., Reusser, K., Rakoczy, K. \& Klieme, E. (2014). One lesson is all you need? Stability of instructional quality across lessons. Learning and Instruction, 31, 2-12. https://doi.org/10.1016/j.learninstruc.2013.12.002

Scheidig, F. (2017). Praxisbezüge arrangieren und analysieren. Der „morphologische Kasten" als Planungs- und Reflexionsinstrument des Praxisbezugs in Studium und Lehre. In M. Weil (Hrsg.), Zukunftslabor Lehrentwicklung. Perspektiven auf Hochschuldidaktik und darüber hinaus (S. 135-159). Münster: Waxmann.

Schröder, H.-J. \& Fischler, H. (2005). Videographierter Unterricht als Hilfsmittel zur Erfassung von Lehrervorstellungen und zur Modifikation von Lehrerhandeln. In M. Wenzel \& H. Stadler (Hrsg.), "Nimm doch mal die Kamera!". Zur Nutzung von Videos in der Lehrerbildung - Beispiele und Empfehlungen aus den Naturwissenschaften (S. 155-170). Münster: Waxmann.

Seidel, T., Blomberg, G. \& Stürmer, K. (2010). „Observer“ - Validierung eines videobasierten Instruments zur Erfassung der professionellen Wahrnehmung von Unterricht. Projekt OBSERVE. In E. Klieme, D. Leutner \& M. Kenk (Hrsg.), Kompetenzmodellierung. Zwischenbilanz des DFG-Schwerpunktprogramms und Perspektiven des Forschungsansatzes (S. 296-306). Weinheim: Beltz.

Seidel, T. \& Thiel, F. (2017). Standards und Trends der videobasierten Lehr-Lernforschung. Zeitschrift für Erziehungswissenschaft, 20 (Supplement 1), 1-21.

Sherin, M. G. \& van Es, E. A. (2009). Effects of Video Club Participation on Teachers' Professional Vision. Journal of Teacher Education, 60 (1), 20-37.

van den Bogert, N., van Bruggen, J., Kostons, D. \& Jochems, W. (2014). First steps into understanding teachers' visual perception of classroom events. Teaching and Teacher Education, 37, 208-216.

Vohle, F. (2013). Relevanz und Referenz: Zur didaktischen Bedeutung situationsgenauer Videokommentare im Hochschulkontext. In G. Reinmann, M. Ebner \& S. Schön (Hrsg.), Hochschuldidaktik im Zeichen von Heterogenität und Vielfalt (S. 165-181). Norderstedt: Books in Demand. 
Falk Scheidig, Dr., Leiter Lehr- und Curriculumsentwicklung an der Pädagogischen Hochschule FHNW. Arbeitsschwerpunkte: Hochschuldidaktik der Lehrpersonenbildung, Erwachsenenbildung

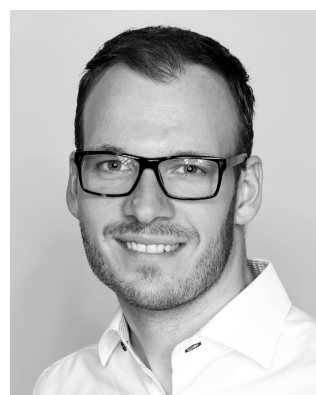

